\title{
Two Miracles of General Relativity
}

\author{
James Read ${ }^{\mathrm{a} *}$, Harvey R. Brown ${ }^{\mathrm{a} \dagger}$, and Dennis Lehmkuhl ${ }^{\mathrm{b} \ddagger}$ \\ ${ }^{a}$ Faculty of Philosophy, University of Oxford, \\ Radcliffe Humanities, Woodstock Road, Oxford OX2 6GG, UK; \\ ${ }^{\mathrm{b}}$ Einstein Papers Project, Caltech M/C 20-7, \\ 1200 East California Blvd., Pasadena, CA 91125, USA.
}

Word count: 11474

Keywords: General relativity; equivalence principle; minimal coupling; dynamical perspective.

\begin{abstract}
We approach the physics of minimal coupling in general relativity, demonstrating that in certain circumstances this leads to (apparent) violations of the strong equivalence principle, which states (roughly) that, in general relativity, the dynamical laws of special relativity can be recovered at a point. We then assess the consequences of this result for the dynamical perspective on relativity, finding that potential difficulties presented by such apparent violations of the strong equivalence principle can be overcome. Next, we draw upon our discussion of the dynamical perspective in order to make explicit two 'miracles' in the foundations of relativity theory. We close by arguing that the above results afford us insights into the nature of special relativity, and its relation to general relativity.
\end{abstract}

*james.read@philosophy.ox.ac.uk

†harvey.brown@philosophy.ox.ac.uk

†lehmkuhl@caltech.edu 


\section{Contents}

1 Introduction $\quad 3$

2 Electromagnetism $\quad 5$

3 Minimal Coupling and the Equivalence Principle 9

4 The Dynamical Perspective $\quad 13$

5 Two Miracles of General Relativity $\quad 18$

6 The Geometrical Perspective $\quad 21$

7 Special Relativity $\quad 24$

8 Conclusions and Outlook 26

A Minimal Coupling and Poincaré Invariance $\quad 27$

B Lorentz Symmetry Breaking $\quad 32$ 


\section{Introduction}

Recently, there has arisen a heightening of interest in the physics community in the coupling of Maxwell electrodynamics to Einstein gravity. For example, the minimal coupling of electromagnetism to gravity leads to curvature terms in second (and higher) order dynamical equations governing non-gravitational fields, written at any point; ${ }^{1}$ however, there also exist different possible coupling schemes, according to which one recovers different dynamical equations at any point. In literature such as $[25,28]$, authors have attempted to elaborate the physical and mathematical details of these different possible coupling schemes.

The purpose of this paper is to pursue a philosophical branch of enquiry into the coupling of electromagnetism to gravity; this field provides fertile ground for foundational insights into both special and general relativity (SR and GR, respectively). A crucial starting point in this regard is that the existence of curvature terms in higher order dynamical equations governing non-gravitational fields, written at any point, indicates violations of the strong equivalence principle (sometimes: Einstein equivalence principle ${ }^{2}$ ). In this paper, we take the basic idea of the priciple to be that (what are typically understood to be) the dynamical laws of SR-i.e. dynamical laws governing matter fields in a fixed Minkowski background, featuring no curvature terms - are recovered in GR at any point. This observation that there exist apparent violations of the strong equivalence principle (made by Eddington as early as 1923 [18, p. 176]) requires a rethinking of the foundations of GR, as this principle is still sometimes asserted to be universally valid in texts on the subject (see e.g. [5, p. 169]).

\footnotetext{
${ }^{1}$ In this paper, we mean by 'matter fields', or 'non-gravitational fields', those for which there exists an associated stress-energy tensor, and by 'gravitational fields' those for which there exists no such stress-energy tensor-this distinction is in the spirit of [35]. In the context of general relativity, this means that the metric field is identified as a gravitational field (see [15] for a proof against the existence of a tensorial expression of such stress-energy), whereas all other fields typically of interest (e.g. Klein-Gordon fields, electromagnetic fields, etc.) are matter fields. There exist subtle issues regarding 'gravitational' stress-energy in general relativity—see e.g. $[27,33,49]$ for related discussion. Note also that this distinction between matter and gravitational fields may break down in the case of other spacetime theories-for example, in Newtonian gravitation theory, it is possible to define a stress-energy tensor associated with the potential $\varphi$, in spite of this field naturally being regarded as 'gravitational' (cf. [16]). Nevertheless, for our purposes, the above distinction will suffice.

${ }^{2}$ See e.g. [10, §IV] , and [46, p. 219]. We distinguish different ways of making this principle precise in $\$ 3.2$. Despite the fact that the principle has been named after Einstein, it should be noted that Einstein himself meant something different when he spoke of the 'equivalence principle'. Though he subscribed both to his definition of the equivalence principle, and to the local validity of SR, he saw the two principles as clearly distinct, as is most clear in [20]. For details of what Einstein calls the 'equivalence principle', see [41]. For these reasons, we use the 'strong equivalence principle' nomenclature in this paper. (For more on different versions of the equivalence principle in general, see [36].)
} 
To illustrate, the details of minimal coupling raise important questions regarding the $d y$ namical perspective on relativity (principally advanced in $[5,8,9]$ ), according to which the metric field in GR, though ontologically distinct from matter fields, acquires its chronogeometric significance via the way it couples to matter fields in accord with the strong equivalence principle, and codifies the symmetries of local dynamical laws. The claim that there exist violations of the strong equivalence principle raises questions over whether this account can still go through. Though the subtleties presented by violations of this principle require us to make slight modifications to the dynamical view in the context of GR, we argue that the problems for the dynamical perspective presented by such cases can be overcome.

Having appreciated the details of such cases, other foundational results follow. In particular, consideration of how the dynamical perspective should be understood in GR in light of these results leads us to identify two crucial foundational aspects-or 'miracles' - in the context of GR. ${ }^{3}$ In turn, this leads to an understanding of the relation of SR to GR which extends the previous notion that dynamical laws in a Minkowski background being recovered in GR at any point characterises this relation-instead, we suggest a liberalised notion of what constitutes a 'special relativistic' theory, according to which the sole defining characteristic of such theories is the Poincaré invariance of dynamical laws governing matter fields. ${ }^{4}$

The structure of this paper is as follows. In $\S 2$, we discuss electromagnetism both in fixed Minkowski spacetime, and in dynamical, generically curved spacetime- the latter theory constructed on the basis of minimal coupling. In $\S 3$, we consider the ramifications of the minimal coupling of electromagnetism to gravity for our understanding of the strong equivalence principle. In $\S 4$, we expound potential problems for the dynamical perspective which arise from this work, showing that ultimately such worries can be overcome. In §5, we highlight two 'miracles' in the foundations of GR. In §6, we raise concerns about the 'geometrical' alternative to the dynamical approach in the context of GR. In $\S 7$, we make some remarks on the nature of SR and its relation to GR. We close in $\S 8$ with conclusions and outlook.

\footnotetext{
${ }^{3}$ By 'miracle', we mean something surprising or puzzling, the explanation of which remains outstanding. Another example of such a 'miracle' in physics would be the proportionality of gravitational and inertial masses in Newtonian mechanics. Just as, in that case, one has to move to a successor theory (namely, GR) to account for such a 'miracle' (cf. [53]), in our view accounting for the two 'miracles of GR' which we specify in this paper will require recourse to considerations from physical theories external to GR. Cf. footnote 46.

${ }^{4}$ The distinction between 'dynamical laws in a Minkowski background' and 'Poincaré-invariant dynamical laws' will be made clear in the body of this paper.
} 


\section{Electromagnetism}

\subsection{Minkowski Spacetime}

Consider Maxwell electrodynamics in a flat, static Minkowski background. In that situation, the metric field $\eta_{a b}$ is a fixed field in the sense of [45, p. 13] (i.e. $\eta_{a b}$ is identically the same in all kinematically possible models), and the Maxwell equations can be written: ${ }^{5}$

$$
\begin{aligned}
F_{; b}^{a b} & =J^{a}, \\
F_{[a b ; c]} & =0,
\end{aligned}
$$

where covariant derivatives are here taken with respect to the torsion-free derivative operator compatible with $\eta_{a b}$ (see e.g. [38, p. 49]); $F_{a b}=-F_{b a}$ is the Faraday tensor; and we define

$$
F^{a b}:=\eta^{a c} \eta^{b d} F_{c d}
$$

with $\eta^{a b}$ the inverse of $\eta_{a b}$. (2.1) can be derived from a variational principle: the standard Lagrangian density for a sourced Maxwell field is given by

$$
\mathcal{L}_{\mathrm{EM}}=-\frac{1}{4} F_{a b} F^{a b}-A_{a} J^{a}
$$

where $A^{a}$ is the electromagnetic vector potential; $A_{a}:=\eta_{a b} A^{b}$; and $J^{a}$ is a source term.

\footnotetext{
${ }^{5}$ Throughout this paper, abstract (i.e. coordinate-independent) indices are written in Latin script beginning $a, b, c, \ldots$; indices in a coordinate basis are written in Greek script; 3 -vector indices in a coordinate basis are written in Latin script beginning $i, j, k, \ldots$; semicolons indicate covariant derivatives; commas indicate partial derivatives; and the Einstein summation convention is used. Round brackets around indices denote symmetrisation over those indices; square brackets around indices denote antisymmetrisation. In addition, we set $\epsilon_{0}=\mu_{0}=c=G_{N}=1$.
} 
Recalling that $F_{a b}$ can be written as

$$
F_{a b}=2 A_{[a ; b]}
$$

and then applying Hamilton's principle with respect to arbitrary variations in the $A_{a}$, we obtain (2.1). (2.2) follows from (2.5). ${ }^{6}$ The stress-energy tensor for the electromagnetic field is ${ }^{7}$

$$
T^{a b}=F_{c}^{a c} F_{c}^{b}-\frac{1}{4} \eta^{a b} F_{c d} F^{c d}
$$

Finally, $F_{a b}$ satisfies a wave equation, which can be derived by manipulation of (2.1) and (2.2):

$$
F_{a b ; c}{ }^{c}=2 J_{[a ; b]}
$$

\subsection{Dynamical Spacetime}

In GR, the metric field $g_{a b}$ is not fixed in all kinematically possible models, and moreover is dynamically coupled to the matter fields via Einstein's equation. Additionally, one need not restrict $a b$ initio the allowed form of the dynamical equations for matter fields (e.g., arbitrary contractions of the matter fields and the curvature tensor $R_{b c d}^{a}$ associated with the torsion free, metric-compatible derivative operator $\nabla_{a}$ are permitted). However, that being said, one often restricts to dynamical equations for non-gravitational fields which are minimally coupled. The prescription for constructing such minimally coupled equations is the following:

Minimally coupled dynamical equations for matter fields in GR are constructed from dynamical equations for matter fields featuring coupling to a fixed Minkowski metric field $\eta_{a b}$ and no curvature terms, by replacing all instances of $\eta_{a b}$ with a

\footnotetext{
${ }^{6}$ If the derivative operator in (2.1) and (2.2) is not assumed to be metric-compatible, then a metric field is only required to write down (2.1), and not (2.2). Cf. appendix A.

${ }^{7}$ Recall that the stress-energy tensor is defined through $T^{a b}=\frac{2}{\sqrt{g}} \frac{\delta S}{\delta g_{a b}}$, where $g$ is the metric determinant, and $S$ is the action to which the matter Lagrangian-here $\mathcal{L}_{\mathrm{EM}}$-is associated.
} 
generic Lorentzian metric field $g_{a b}$, and replacing all instances of the torsion-free derivative operator compatible with $\eta_{a b}$ with the torsion-free derivative operator compatible with $g_{a b}$.

In the case of electromagnetism in the GR context, if one couples the $F_{a b}$ to $g_{a b}$ via

$$
\begin{gathered}
F_{; b}^{a b}=J^{a}, \\
F_{[a b ; c]}=0,
\end{gathered}
$$

and

$$
G^{a b}:=R^{a b}-\frac{1}{2} g^{a b} R=8 \pi T^{a b}
$$

then one obtains Maxwell electrodynamics minimally coupled to Einstein gravity. Here, $T^{a b}$ is the stress-energy tensor of $F_{a b} ; g^{a b}$ is the inverse of $g_{a b}$; indices are lowered with respect to $g_{a b}$ and raised with respect to $g^{a b}$; the derivative operator is torsion-free and metric-compatible; and the Ricci tensor $R_{a b}:=R_{a c b}^{c}$ and Ricci scalar $R:=g^{a b} R_{a b}$ are those associated to that derivative operator. (2.8) and (2.9) are Maxwell's equations in this (dynamical and generically curved) spacetime; and (2.10) is Einstein's equation. In this case, $T^{a b}$ on the right hand side of $(2.10)$ reads $^{8}$

$$
T^{a b}=F^{a c} F_{c}^{b}-\frac{1}{4} g^{a b} F_{c d} F^{c d}
$$

In this minimally-coupled Maxwell-Einstein system, the wave equation for $F_{a b}$ becomes,

\footnotetext{
${ }^{8}$ More generally, $T^{a b}$ is the stress-energy tensor associated to all matter fields in the theory.
} 
after using (2.8), (2.9), and Ricci-like identities, ${ }^{9}$

$$
F_{a b ; c}^{c}=2\left(F_{[b}^{e} R_{a] e}-R_{a b c d} F^{c d}+J_{[a ; b]}\right) .
$$

In (2.7), the two curvature terms on the right hand side of (2.12) were not present, as the metric field was fixed to be $\eta_{a b}$ - a fortiori flat—in all kinematically possible models. Dropping this assumption leads to the generalised form (2.12).

There is an important feature of second-order equations such as (2.12), valid in minimallycoupled Maxwell-Einstein dynamics. To see this, first recall that in a coordinate basis $\left\{e_{\mu}\right\}$, the connection components $\Gamma_{\nu \rho}^{\mu}$ associated to a derivative operator $\nabla_{a}$ are defined by $\nabla_{\rho} e_{\nu}=$ $\Gamma_{\nu \rho}^{\mu} e_{\mu}$. Then, where $M$ is the spacetime manifold, at any $p \in M$ we can choose normal coordinates, such that $\Gamma_{(\nu \rho)}^{\mu}(p)=0$ in those coordinates; for a torsion-free derivative operator, we can in fact choose normal coordinates such that $\Gamma_{\nu \rho}^{\mu}(p)=0$. (Note that the connection components away from $p$ will in general not vanish.) If the unique torsion-free, metric compatible derivative operator is used, then in normal coordinates we also have $g_{\mu \nu, \rho}(p)=0$, and we may find a subclass of normal coordinates at $p$ such that $g_{\mu \nu}(p)=\operatorname{diag}(-1,1,1,1) \cdot{ }^{10}$ Since $g_{\mu \nu}(p)$ takes this diagonal form—-preserved under Poincaré transformations—one might write $g_{\mu \nu}(p)=\eta_{\mu \nu}$ (cf. e.g. [40, p. 1055]). This notwithstanding, however, any claim to the effect that the metric field 'reduces' to the Minkowski metric at $p$ in normal coordinates should be met with suspicion - for in general, second (and higher) order derivatives of the metric field do not vanish at $p$, in these coordinates. This point will be of importance in what follows.

In normal coordinates at $p$, connection components vanish—and elements of the class of frames in which such is the case, and in which $g_{\mu \nu}(p)=\operatorname{diag}(-1,1,1,1)$, are related to one another by Poincaré transformations. Moreover, first order, minimally coupled dynamical equations such as (2.8) and (2.9) do not feature curvature terms at $p$. In this way, first order, minimally coupled dynamical equations recover their 'Minkowski background' form at $p$. However, this is only generically true of first order equations; equations that are second or

\footnotetext{
${ }^{9}$ I.e. identities of the form $\left(\nabla_{c} \nabla_{d}-\nabla_{d} \nabla_{c}\right) T_{b_{1} \ldots b_{l}}^{a_{1} \ldots a_{k}}=R^{a_{1}}{ }_{e c d} T_{b_{1} \ldots b_{l}}^{e \ldots a_{k}}+\cdots+R^{a_{k}}{ }_{e c d} T_{b_{1} \ldots b_{l}}^{a_{1} \ldots e}$ $R_{b_{1} c d}^{e} T^{a_{1} \ldots a_{k}}{ }_{e \ldots b_{l}}-\cdots-R_{b_{l} c d}^{e} T_{b_{1} \ldots e}^{a_{1} \ldots a_{k}}$.

${ }^{10}$ We say here 'a subclass', for the conditions on normal coordiantes at $p$ are preserved under all affine transformations, whereas the condition $g_{\mu \nu}(p)=\operatorname{diag}(-1,1,1,1)$ is preserved only under a subclass of such transformations-viz., Poincaré transformations. In the remainder of this paper, we invariably focus upon those normal coordinates in which this diagonalisation condition holds, without explicit qualification.
} 
higher order may contain curvature pieces and higher order covariant derivatives-consider, for example, (2.12) versus (2.7). Since curvature is represented by the Riemann tensor, this object cannot be made to vanish at $p$. This implies that in GR it is not always the case that laws recover their original 'Minkowski background' forms at a point. Another way to see this is to note that by expanding out the curvature and covariant derivative terms in such equations, we obtain terms containing derivatives of connection coefficients, which in general cannot be made to vanish at $p$ in normal coordinates. ${ }^{11}$

\section{Minimal Coupling and the Equivalence Principle}

\subsection{Local Dynamical Equations}

What should one make of the observation that minimal coupling yields second order equations such as (2.12) containing curvature terms which do not vanish at a point? To answer this question, it is useful to distinguish two forms that the dynamical laws for non-gravitational fields might take in normal coordinates at any given point $p \in M$ in GR: (a) Poincaré invariant, with no terms containing the Riemann tensor or its contractions; or (b) Poincare invariant simpliciter. In $\S 2$, we saw that minimal coupling yields violations of the claim that, at any $p \in M$, dynamical laws take a Poincaré invariant form with no terms featuring the Riemann tensor or its contractions (i.e., form (a)). However, this does not necessarily imply violations of the analogous claim regarding such laws taking, at any $p \in M$, a Poincaré invariant form simpliciter (i.e., form (b)).

One important point to make regarding minimal coupling is the following: although a certain form of dynamical law (i.e. those laws featuring coupling to a fixed Minkowski metric field, and containing no curvature terms) is used to determine the class of minimally coupled dynamical laws for non-gravitational fields in GR, there exists no a priori restriction on the form that these general relativistic, minimally coupled equations take locally, at any $p \in M-$ in particular, there exists no restriction that we recover locally the dynamical laws from which

\footnotetext{
${ }^{11} \mathrm{Cf}$. [10, §IV]. The present discussion goes further than that paper in several respects-for example, in explicitly delineating precisifications of the strong equivalence principle which are in tension with the above results.
} 
we began. That said, it is straightforward to show that such minimally coupled dynamical equations in GR take a Poincaré invariant form-but not necessarily a form in which terms containing the Riemann tensor or its contractions vanish - at any $p \in M .{ }^{12}$ In this way, one can consider (minimal) coupling to matter to restrict the local symmetry group of the dynamical equations of GR to the Poincaré group.

Some further words on the ambiguity of the application of minimal coupling are in order. Clearly, the minimal coupling prescription presented in $\$ 2.2$ does not specify the dynamical equations to which the procedure is to be applied. Though in $\S 2.2$, the procedure was applied to (2.1) and (2.2), yielding (respectively) (2.8) and (2.9), which in turn were used to construct (2.12), it is not the case that, were the minimal coupling procedure applied directly to (2.7), (2.12) would have been obtained. Thus, there is a sense in which the minimal coupling prescription is ambiguous. ${ }^{13}$ This is in line with Goenner's observation that "such a procedure is unique only for first-order partial differential equations" [24, p. 866].

A related point here is the following. One might think that it is no surprise that equations such as (2.12) do not reduce to (2.7) (i.e., to a Poincaré invariant form, with no curvature terms) in normal coordinates at any $p \in M$, for (2.12) was not obtained directly via minimal coupling. This thought, however, is ultimately by-the-by, for even if minimal coupling were applied directly to (2.7), to obtain

$$
F_{a b ; c}{ }^{c}=2 J_{[a ; b]},
$$

where now index contraction is taken with respect to a generic Lorentzian $g_{a b}$ satisfying (2.10), and the unique torsion free derivative operator $\nabla_{a}$ compatible with $g_{a b}$ is used, (3.1) would still not take at any $p \in M$ and in normal coordinates a Poincaré invariant form with no curvature terms - the reason being that, in a coordinate basis, (3.1) features derivatives of connection components, which cannot be made to vanish in normal coordinates.

\footnotetext{
${ }^{12} \mathrm{Cf}$. appendix A. The work presented in that appendix also brings out when such local dynamical equations are invariant under a broader symmetry group than the Poincare group. The reader's attention should also be drawn to $\S 6$ and appendix B, in which cases are presented in which dynamical equations for matter fields take a yet simpler form (in the sense that further terms in these equations vanish) in a class of frames related by a subgroup of the Poincaré group.

${ }^{13}$ For discussion related to this point, see $[40, \S 16.3]$.
} 


\subsection{The Strong Equivalence Principle}

The work presented in $\S 2$ has important ramifications for the strong equivalence principle. We here introduce two distinct formulations of this principle, which we call EP1 and EP2: ${ }^{14}$

EP1: The dynamical equations for non-gravitational fields reduce to a Poincaré invariant form, with no terms featuring the Riemann tensor or its contractions, at any $p \in M$.

EP2: The dynamical equations for non-gravitational fields reduce to a Poincaré invariant form at any $p \in M$.

The results of $\S 2$ indicate that EP1 is incompatible with minimal coupling for higherorder dynamical equations like (2.12). Claims to the contrary can, however, be found in the foundational literature; consider for example the following quote, given at [5, pp. 170-171]:

[Minimal coupling involves the] claim that the matter fields do not couple to the Riemann curvature tensor or its contractions. Recall that in SR, inertial frames are global, which implies that the curvature vanishes everywhere, and hence trivially makes no appearance in the laws of physical interactions. This feature is now absorbed into GR in the requisite local context.

Since terms featuring in (2.12) contain the Riemann tensor, these cannot be made to vanish at a point; this is in manifest contradiction with the above quote. In fact, second order equations such as (2.12) constitute a straightforward counterexample to any claims to the effect that EP1 may universally be regarded as holding when dynamical equations for matter fields are constructed via minimal coupling. Note, however, that insofar as curvature terms may be ignored at $p \in M$, EP1 may be regarded as holding at $p$. Whether this is the case will principally depend upon the strength of curvature effects relative to the experimental apparata available; if one's experimental apparata are insensitive to such effects, then the principle may be regarded as holding approximately at $p$ for the matter field measured by those apparata—see below for further discussion. By contrast to EP1, note that curvature couplings in higher-order

\footnotetext{
${ }^{14}$ Sometimes, EP1 and EP2 are referred to as 'pointy' versions of the strong equivalence principle-for discussion, see $[23,31,42]$.
} 
minimally coupled dynamical equations for matter fields do pose problems for the validity of EP2 in the context of matter fields obeying such dynamical equations-indeed, the results of $\S$ A demonstrate that there exists no tension between EP2 and minimal coupling.

Recall now the discussion of the strong equivalence principle at $\left[5\right.$, p. 169]: ${ }^{15}$

There exists in a neighbourhood of each event preferred coordinates, called locally inertial at that event. For each fundamental non-gravitational interaction, to the extent that tidal gravitational forces can be ignored, the laws governing the interaction find their simplest form in these coordinates. This is their special relativistic form, independent of spacetime location.

To understand this quote, it is useful to extend EP1 and EP2 to the neighbourhood of any $p \in M:$

EP1': The dynamical equations for non-gravitational fields reduce to a Poincaré invariant form, with no terms featuring the Riemann tensor or its contractions, in a neighbourhood of any $p \in M$.

EP2': The dynamical equations for non-gravitational fields reduce to a Poincaré invariant form in a neighbourhood of any $p \in M$.

As with EP1, whether EP1' holds approximately will principally depend upon the strength of curvature effects relative to the experimental apparatus available. (Arguably, however, such assumptions are more plausible in the context of EP1' than of EP1, since experimental apparata always occupy some extended region of spacetime. ${ }^{16}$ In this case, however, the size of the neighbourhood is also relevant: in a larger neighbourhood of $p$, one is more likely to be able to detect curvature effects with one's experimental apparatus-in which case, EP1' will not hold as an approximate principle. To illustrate, consider Pound-Rebka experiments to detect gravitational redshift. ${ }^{17}$ The fact that these experimental apparata are not sensitive to curvature effects in their neighbourhood means that EP1' can be regarded as holding in

\footnotetext{
${ }^{15}$ Other similar presentations of the strong equivalence principle can be found in e.g. [31, §3.4] and [32, p. 874].

${ }^{16}$ In this regard, cf. [43, §1.9], and discussion at [5, p. 170] and [32, p. 875].

${ }^{17}$ For the original Pound-Rebka paper, see [47].
} 
that neighbourhood for the fields that those apparata measure; in light of this, a flat spacetime account of the Pound-Rebka results can be offered. For further details, see [10, §III]. (Though EP2' is principally introduced by analogy with EP2, it is important to note that, like EP1', this principle also holds only approximately in a neighbourhood for minimally coupled dynamical equations_-for it relies upon the metric field $g_{a b}$ to which the matter fields couple being approximately Minkowskian in a neighbourhood.)

Clearly, it is important that one be precise about what is meant by 'tidal gravitational forces' in quotations such as that above. In the context of dynamical equations for nongravitational fields in the neighbourhood of some $p \in M$, we understand 'terms representing tidal forces' to mean terms in those equations featuring the Riemann tensor or its contractions. As a result, in contexts in which terms representing tidal forces can be ignored, EP1' and EP2' hold.

\section{The Dynamical Perspective}

With this understanding of the limitations to the holding of EP1 in hand, we now consider potential ramifications of this result for positions in the foundations of relativity theory. To that end, we assess in this section the consequences of the above work for the dynamical perspective on relativity, as presented in $[5,8,9]$. We argue that difficulties raised by violations of EP1 do not present damning problems for this view in the context of GR.

\subsection{Explanation and Codification}

To begin our discussion, we must clarify what is meant by the dynamical perspective on relativity. We first focus specifically on the case of $S R$, and distinguish two positions on the nature of the Minkowski metric field:

(A) The Minkowski metric field is an ontologically distinct and primitive entity; its presence can explain certain facts about the dynamical laws governing matter fields (namely, the fact that these laws are Poincaré invariant). 
(B) The Minkowski metric field is not an ontologically distinct and primitive entity; rather, it is a codification of certain facts about the dynamical laws governing matter fields (namely, the fact that these laws are Poincaré invariant).

To endorse (B) is to endorse the dynamical perspective on relativity; the orthodox line is (A). ${ }^{18}$ In advocacy of the (A)-view, Maudlin writes: ${ }^{19}$

If we accept that in a vacuum there is no physical structure, except for the structure of space-time itself, then the behaviour of light in a vacuum implies that the geometry of spacetime alone determines the trajectory of the light rays. That is, given any point in the space-time $p$, the structure of space-time ought to fix where light emitted from that $p$ (in any possible direction) will go. [39, p. 68] (Emphasis in original.)

... the Minkowski geometry takes exactly the same form described in [any] Lorentz coordinate system (by the symmetry of Minkowski spacetime), and the laws of physics take exactly the same coordinate-based form when stated in a coordinatebased language in any Lorentz coordinate system (because the laws can only advert to the Minkowski geometry, and it has the same coordinate-based description). ${ }^{20}[39$, pp. $117-118]$

By contrast, the (B)-view (principally advocated by Brown and Pooley—see [5, 8, 9]) is ex-

\footnotetext{
${ }^{18}$ Acuña [1] has recently argued that the debate between (A) and (B) is misguided. In particular, he argues that neither Minkowski spacetime nor Poincaré invariance is to be taken as explanatory and/or ontologically primary over the respective other, but that the connection between the two is one of bidirectional analyticity: Spacetime is Minkowskian just in case the laws of physics are Poincare invariant. We concede that this is a defensible position (at least prima facie), even though Acuña does not show that it is superior to either (A) or (B). Moreover, his position does not have a natural extension to GR; in the following, we shall discuss such an extension of (B).

${ }^{19}$ We take Maudlin's speaking of Minkowski spacetime in vacuo to indicate his commitment to the view that the metric field of SR is primitive, and ontologically autonomous of matter fields. Other advocates of the (A)view arguably include e.g. Friedman [22]. A related view is that of Janssen [2,30], according to whom one makes a 'common origin inference' from the Poincaré invariance of the dynamical laws to Minkowski spacetimeunderstood as an expression of the universal Poincaré invariance of all dynamical laws. On this position, the Minkowski metric field is not necessarily an ontologically autonomous entity. For further discussion as to how Janssen's views align with the (A)- and (B)-views, see [1,48].

${ }^{20}$ In this connection, note also that Einstein, after developing GR, came to view the structure of Minkowski spacetime in SR as affording an explanation of the inertial motion of force-free bodies, and thus as violating the action-reaction principle. For a critical analysis of this development in Einstein's thinking, see [7].
} 
pressed in passages such as the following: ${ }^{21}$

The appropriate structure is Minkowski geometry precisely because the laws of the non-gravitational interactions are Lorentz covariant. [5, p. 133]

The relative merits of (A) and (B) in the context of SR have been widely discussed, and will not be repeated here. Instead, we observe how the debate between these two camps changes on moving to GR. The first key difference in the case of GR is that the advocate of (B) concedes that the metric field in this context is an autonomous agent, ontologically distinct from the matter fields of the theory. ${ }^{22}$ Hence, for the extensions to GR of both (A) and (B), the metric field is not reducible to properties of the matter fields.

Though advocates of (A) and (B) agree on the ontological autonomy of the metric field in GR, they may disagree on its relation to chronogeometry. For the latter, the dynamics of the metric field tell us that it is 'just another field': "Nothing in the form of the equations per se indicates that $g_{a b}$ is the metric of space-time, rather than a $(0,2)$ symmetric tensor which is assumed to be non-singular" $\left[5\right.$, p. 160]. ${ }^{23}$ How, then, does the metric field attain its chronogeometric significance in GR? For the proponent of (B), the metric field "earns its spurs by way of the strong equivalence principle" [5, p. 151]. The reasoning here can be stated explicitly as follows:

1. In the neighbourhood of any $p \in M$, assuming that one's experimental apparata are such that terms featuring the Riemann tensor or its contractions can be ignored, we recover EP1', for the fields measured by those apparata. ${ }^{24}$

\footnotetext{
${ }^{21}$ Note that what Brown calls 'Lorentz covariance' here is what we call 'Poincare invariance' above. Related to this, it is worth noting that arguably the essence of SR is more closely tied to Lorentz- than Poincaréinvariance, for both pre-relativistic and relativistic dynamical laws governing matter fields are locally invariant under translations-it is only the linear part of the class of affine transformations under which such laws are invariant that changed on the transition to relativistic physics.

${ }^{22}$ To claim that the metric field is reducible to the matter fields in GR is to endorse a certain form of relationism about the metric field; there are profound difficulties with implementing this programme in GR. An obvious illustration of this difficulty can be found in the existence of vacuum solutions in the theory.

${ }^{23}$ Notation in this quotation has been modified for consistency with the present paper. For discussion of coordinate-dependent versus -independent approaches in the foundations of spacetime theories, see [52].

${ }^{24}$ Note that this invocation of approximation means that, strictly speaking, we are dealing with the approximate chronogeometric significance of the metric field in GR. Our thanks to an anonymous referee for pushing us on this point.
} 
2. In addition, in such a scenario the dynamical metric field of GR manifests Poincaré symmetries - for in this case, higher derivatives of the metric field can be regarded as vanishing. ${ }^{25}$

3. The symmetries of the dynamical laws governing non-gravitational fields in the appropriate local neighbourhood of $p$ therefore coincide with the symmetries of the dynamical metric field in this neighbourhood. ${ }^{26}$

4. As a result, inertially moving, stable physical rods and clocks survey this primordial metric field, and so afford it its chronogeometric significance, in the neighbourhood of $p .^{27}$

In summary, the point may be put as follows:

It is because of minimal coupling and local Lorentz covariance that rods and clocks, built out of the matter fields which display that symmetry, behave as if they were reading aspects of the metric field and in so doing confer on [the metric] field a geometrical meaning. [5, p. 176]

It is worth making some comments on the (B)-view. First, the advocate of this approach errs insofar as she universally endorses EP1 in conjunction with minimal coupling, for we have seen cases in which the minimal coupling prescription applied to certain dynamical laws

\footnotetext{
${ }^{25}$ By imposing the condition that terms representing tidal forces may be ignored, derivatives of the metric field may be ignored, so this field may indeed be treated as the Minkowski metric field in the relevant local vicinity of $p$. Note that thus far we have understood 'symmetries' to mean dynamical symmetries-i.e. transformations upon dynamical equations which leave their form invariant. When speaking of the (local) symmetries of the metric field, we are concerned with spacetime symmetries, which Pooley defines as "groups of transformations that preserve spatiotemporal structure (as encoded in coordinate systems)" [44, §3.1]. (Clearly, the advocate of the dynamical approach may resist the ab initio identification of the metric field with 'spacetime'; this, however, does not affect the above presentation of the dynamical view.)

${ }^{26} \mathrm{On}$ this point, an anonymous referee has raised the question, "Since this notion of symmetry coincidence is symmetrical, why not say that the metric field surveys rods and clocks?" Our response is that the asymmetry is broken through the fact that stable rods and clocks can be built from matter fields (though cf. footnote 27), which then read off intervals as given by the metric field-but it is not (it appears) the case that stable rods and clocks can be built from the metric field, which then survey matter fields in the theory. We concede, however, that this latter possibility is of interest, and deserves further exploration.

${ }^{27}$ Note that the local coincidence of metric and dynamical symmetries, as delivered by the strong equivalence principle, is only a necessary condition for the metric field to have chronogeometric significance tout court. For example, as flagged above, the existence of stable rods and clocks built from matter fields is an additional, non-trivial assumption.
} 
implies the negation of EP1 (cf. §3). This tension is not necessarily problematic, however, for what is sufficient for the (B)-view to go through in the general relativistic context (for the reasons detailed above) is that, in an appropriate neighbourhood of any $p \in M$, terms featuring the Riemann tensor or its contractions may be ignored-so that EP1' holds in this neighbourhood. ${ }^{28}$

What is a plausible (A)-type counterpart to the (B)-view in the context of GR? We take this to be the following: the metric field has a primitive connection to spacetime geometry, and in the regime in which terms featuring the Riemann tensor or its contractions may be ignored, the dynamical laws governing non-gravitational fields in a suitable neighbourhood of any point $p \in M$ are constrained to be invariant with respect to the local symmetries of this field in the same manner as for the (A)-story in the context of SR. Hence, the existence of the Lorentzian metric field explains the form of the local dynamical laws in the theory. ${ }^{29}$

\subsection{The Equivalence Principle and the Dynamical Perspective}

Henceforth, we take (A) and (B) to refer to the above formulations of these views in the context of GR. How, then, do these fare in light of $\S 3$ ? As we have seen, the advocate of the (B)-view makes an inconsistent move insofar as she assumes the universal validity of EP1 alongside minimal coupling. There is, however, a straightforward fix available: she may simply maintain that, to the extent that terms featuring the Riemann tensor or its contractions may be ignored, EP1' holds, and the metric field takes the form of the Minkowski metric in the relevant neighbourhood. These latter results suffice for the coherence of the dynamical approach in the context of GR.

The Poincaré invariance of local dynamical laws written in normal coordinates at any $p \in M$ merits further discussion. In particular, one might worry that there exists a tension between (I) the fact that such laws are invariant under translations, and (II) the fact that these laws generically do not hold at some other $q \in M$ in the neighbourhood of $p$ (now setting aside

\footnotetext{
${ }^{28}$ Strictly, the story told by the advocate of the dynamical perspective regarding the chronogeometricity of the metric field can go through at a point $p \in M$, so long as tidal forces may be ignored at that point (so that EP1 holds approximately at that point). However, since (as discussed above) experimental apparata always occupy some extended region of spacetime, it is more natural to focus upon a neighbourhood of any $p \in M$, and thereby upon the approximate validity of EP1' for the fields measured by those apparata.

${ }^{29}$ We raise in $\S 6$ some worries regarding this account.
} 
the dropping of terms representing 'tidal forces'). To see this, suppose that one takes an active interpretation of such translations. In that case, one appears to find that the dynamical laws at $p$ invariably also hold at $q-$ a contradiction. Tension here can be resolved in the following way: one should not take an active interpretation of such translations upon the dynamical equations at $p .{ }^{30}$ The reason for this is that, in writing the minimally coupled dynamical equations of GR at $p$ in normal coordinates, information encoded in connection coefficients is not retained. ${ }^{31}$ An actively-interpreted translation upon these equations at $p$ would tell us that connection coefficients also vanish at $q$, in the same coordinate system-but we have prior knowledge that this is not the case. Hence, one should not interpret actively translations performed upon such dynamical equations, once written in normal coordinates at any $p \in$ $M^{32}$

\section{Two Miracles of General Relativity}

In response to claims to the effect that metric structure explains the symmetries of the equations governing non-gravitational fields, and in particular the fact that they are all Poincaré invariant, the advocate of (B) may reply that such an explanation is "question begging" [5, p. 139]. Regardless of which side one endorses in this debate, however, the fact that all such dynamical equations manifest the same symmetries is at least an a priori mystery; one might call it the first miracle of relativity (MR1):

MR1: All non-gravitational interactions are locally governed by Poincaré invariant dynamical laws.

\footnotetext{
${ }^{30}$ Here, there are parallels with merely formal symmetries in the case of quantum mechanics, which admit of no active interpretation-see $[6, \S 3.1]$.

${ }^{31}$ Note that this is due to the mathematical fact that connection components vanish at $p$ in normal coordinates; no approximations are invoked in this discussion.

${ }^{32}$ This point is worth stressing, in order to avoid certain confusions. A metric field's being Minkowskian is a global rather than a local property of a manifold, for by definition all Minkowski metric fields are geodesically complete (cf. footnote 45). Thus, geometrically speaking, it is not clear how one can define 'local' Poincaré transformations, as discussed above - since from this point of view, such transformations are usually understood to be the isometries generated by the Killing vector fields on Minkowski spacetime. The resolution is to view the Poincaré transformations discussed above as passive only-their action is not on spacetime points at all, but rather on the chart space, i.e. the codomain of the coordinate charts. Very many thanks to an anonymous referee for helping us to clarify considerably these matters.
} 
MR1 holds in SR: it tells us that the dynamical laws governing all matter fields are Poincaré invariant; the advocate of the dynamical view (B) takes this to be a brute fact; the advocate of the geometrical view (A) attempts to rationalise this by appeal to Minkowski spacetime. ${ }^{33}$ The fact that MR1 holds has been discussed in [2,5], and coheres well with the later thinking of Einstein: "The content of the restricted relativity theory can accordingly be summarized in one sentence: all natural laws must be so conditioned that they are covariant with respect to Lorentz transformations" [21, p. 329]. MR1 still obtains in the neighbourhood of any $p \in M$ in GR. In addition to MR1, however, the work of $\S 4$ makes plausible that there exists in the GR context a second miracle of relativity (MR2):

MR2: The Poincaré symmetries of the dynamical laws governing non-gravitational fields in the neighbourhood of any point $p \in M$ coincide-in the regime in which terms featuring the Riemann tensor or its contractions may be ignored-with the symmetries of the dynamical metric field in that neighbourhood. ${ }^{34}$

In GR, there exists an ontologically autonomous ('primordial') metric field, and this leads to MR2: why is it—assuming that terms representing tidal forces can be ignored-that the symmetries of the dynamical laws governing non-gravitational fields in a suitable neighbourhood of any $p \in M$ coincide with those of the primordial metric field in that neighbourhood? Again, the advocate of the dynamical view (B) may postulate this as a brute fact. By contrast, the advocate of the geometrical view (A) may attempt to argue that the ontologically primitive metric field explains the form of the dynamical laws governing matter fields; however, as in $\mathrm{SR}$, she faces an outstanding burden to delineate how this is supposed to work. ${ }^{35}$

One thing that the advocate of (A) may say here is the following: Minimally coupled

\footnotetext{
${ }^{33}$ On MR1, David Wallace has posed to us the following question: "Why should one think it a miracle that the intersection of the symmetry group of all the dynamical laws governing matter fields is the intersection of the symmetry group of all the dynamical laws governing matter fields?" To this, we respond as follows: the larger this intersection, the bigger the miracle. The fact that this intersection (and indeed also the union of the symmetry group of all dynamical laws governing matter fields) is the Poincaré group certainly qualifies, in our view, as 'miraculous', in the sense of footnote 3.

${ }^{34}$ Another way to put MR2 would be the following: the signature of the metric field which codifies the local symmetries of the dynamical laws governing matter fields (cf. [11, §5]) coincides with the signature of the dynamical metric field appearing in Einstein's equation.

${ }^{35}$ Even supposing that an explanation can be found for MR2, there exist further questions in this vicinity. For example, why (with the programme of Callender in mind-cf. [12]) should the lobes of the lightcone structure designated as timelike by (a) the primordial metric field, and (b) the metric field which codifies the symmetries of the local dynamical laws, coincide? Our thanks to an anonymous referee for useful comments on this point.
} 
dynamical laws in GR feature the metric field $g_{a b}$; as we have seen, the presence of (or rather, the coupling to) this metric constrains the local form in the neighbourhood of any $p \in M$ of the dynamical laws of those fields to which it couples. Consequently, the symmetries of the local dynamical laws must coincide with the symmetries of the metric field. This argument misses the point, however, for the very issue in question is why the dynamical laws governing matter fields take such a form-rather than another, with different local symmetry properties. In other words: why this particular coupling? This is the essence of MR2, which remains untouched by such arguments.

If one could argue that what had previously been regarded as the ontologically independent metric field of GR was in fact reducible to a codification of symmetries of the dynamical equations of matter fields (as with the (B)-story in SR), then this would provide an explanation for MR2. Indeed, one might think there are hints that this can be achieved: vacuum GR is essentially an affine theory, in the sense that the field equation of vacuum GR, $R_{a b}=0$, only require an affine connection to be defined, as the Riemann and the Ricci tensor can be defined in terms of the connection only, without any need to refer to a metric tensor. On the other hand, we do need a metric tensor to define the Ricci scalar that, together with the Ricci tensor, makes up the Einstein tensor that forms the left-hand side of the full Einstein equations. ${ }^{36}$ Hence, one can go a long way in GR without defining a metric field-but as soon as one seeks to treat gravitational fields in the presence of matter, one must introduce a metric field. ${ }^{37}$

Note, however, that with only affine structure at one's disposal, there is no way to distinguish between four-dimensional space and four-dimensional spacetime. The reason is that one needs conformal or metric structure to distinguish between spatial and temporal dimensions, manifested in the signature of the metric (or of the conformal equivalence class of metrics). Thus, the claim that vacuum GR is essentially an affine theory needs to be taken with a grain of salt: we still need to obtain a metric or an equivalence class of metrics to make it a spacetime theory. Consequently, one must take even vacuum GR, understood as a theory of spacetime, to require metric structure, thereby raising potential obstacles for this move to explain MR2. ${ }^{38}$

\footnotetext{
${ }^{36}$ The reason is that while the Ricci scalar can be obtained by contracting the one upper and one of the three lower indices of the Riemann tensor, in order to obtain the Ricci scalar we first need to raise one index of the Ricci tensor using the metric. This also means that we cannot define the standard Einstein-Hilbert Lagrangian without a metric.

${ }^{37}$ This coheres with the results of [35], that in order to define the stress-energy tensor of generic material systems, a metric tensor must be in place. We now find that both sides of the full Einstein equations require a metric in order to be well-defined.

${ }^{38}$ For presentations of GR in which affine structure is introduced before metric structure, see $[38,40,51]$
} 
Alternatively, one might seek to account for MR2 by appeal to a particular form of the relativity principle, which states that the laws of physics take the same form in all inertial frames of reference. Recall that normal coordinates at any $p \in M$ are such that $g_{\mu \nu, \rho}(p)=0$, and one may impose the further restriction that $g_{\mu \nu}(p)=\operatorname{diag}(-1,1,1,1)$. It is plausible to regard such frames as inertial, since connection coefficients vanish therein; this means-via the geodesic equation - that motions of test bodies take their simplest form in such frames, insofar as they follow Newtonian inertial trajectories. Now consider a case in which nongravitational fields governed by Galilean-invariant dynamical laws are coupled-via their associated stress-energy tensor-to Einstein's equation in which the curvature tensor is associated (via the unique torsion free, metric-compatible connection) to a Lorentzian metric field, $g_{a b}$. Suppose, for a given $p \in M$, that there exists a normal coordinate system in which the Galilean-invariant dynamical laws take their simplest form. Now transform to a new coordinate system, via a Lorentz boost. Though this new coordinate system is still normal-and so still inertial-the Galilean-invariant laws do not take the same form in this frame. Thus, this form of the relativity principle is violated. If one holds this principle to be sacrosanct, then one may, therefore, be able to account for MR2. Note, however, that this again merely pushes the problem back, for one may ask: why such a relativity principle?

\section{The Geometrical Perspective}

The situation presented thus far stands as follows. (I): Minimal coupling implies EP2, but does not in general imply EP1 (pace e.g. [5, ch. 9]). (II): When terms featuring the Riemann tensor or its contractions in local dynamical equations for non-gravitational fields can be ignored, EP1' holds in an appropriate neighbourhood of any $p \in M$. (III): Though philosophical positions such as the (B)-view are presented as being interwoven with claims regarding the compatibility of the universal validity of EP1 and minimal coupling (see e.g. [5, ch. 9]), such claims may be excised from the position-for which suffices the approximate validity of EP1'. (IV): Some justification for restricting the allowed class of dynamical equations for non-gravitational fields in GR, or of the relativity principle, would suffice as an account of MR1 and MR2.

though note that, in such sources, this order of presentation is often chosen for pedagogical reasons, rather than to explore the extent to which it is possible to do GR with only affine structure, as is our concern here. 
By analogy with the case of SR, the advocate of the (A)-view is likely to claim that the sheer fact that non-gravitational fields are situated in a spacetime manifesting (under the appropriate conditions, i.e. when terms featuring the Riemann tensor or its contractions may be ignored) local Poincaré invariance in the neighbourhood of any $p \in M$ explains the local symmetries of their associated dynamical laws for matter — and thereby explains MR1 and MR2. In our view, however, this is not correct. In this section, we present two interestingly distinct problem cases, in which the reasoning central to the (A)-view appears insufficient to account for the dynamical behaviour of matter fields. These two cases are the Jacobson-Mattingly theory on the one hand, and certain bimetric theories on the other. ${ }^{39}$

In the former (presented in e.g. $[14,29]^{40}$ ), the action for a coupled Einstein-Maxwell system is augmented with an additional term (via a Lagrange multiplier field $\lambda$ ), imposing (as a field equation, via variation with respect to $\lambda$ ) that the vector potential $A^{a}$ be locally timelike: ${ }^{41}$

$$
S_{\mathrm{JM}}\left[g_{a b}, A^{a}, \lambda\right]=\int d^{4} x \sqrt{-g}\left(R-\frac{1}{4} F^{a b} F_{a b}+\lambda\left(g_{a b} A^{a} A^{b}-1\right)\right) .
$$

The imposition of this Lagrange multiplier term results in a violation of the relativity principle - though in a subtly different form to that presented in $\S 5$. The form of the relativity principle that is violated by the Jacobson-Mattingly theory is the following: dynamical laws for non-gravitational fields take their simplest form in all inertial frames. (Here, the 'simplest' form of a dynamical equation is to be understood as the form in which the greatest number of terms vanish-cf. appendix B.) The reason that this version of the relativity principle is violated is that the Lagrange multiplier term in (6.1) picks out a preferred (timelike) direction in the neighbourhood of any $p \in M$; dynamical equations written in the subclass of inertial

\footnotetext{
${ }^{39}$ On our appeal to these theories, an anonymous referee has raised the following objection: why is the fact that the (A)-view fails in such counterfactual scenarios, in which e.g. the Jacobson-Mattingly theory holds, of any relevance? The reason is that the (A)-view postulates a strong modal constraint: all matter fields must be such that they 'advert' to all and only the designated metric structure, in the sense that the dynamical laws governing matter fields manifest (locally) the symmetries of that metric structure. Scenarios such as those discussed in this section demonstrate that this need not be the case. Now, we leave open the possibility that there exist viable refinements of the (A)-view, which do not postulate such strong modal constraints-indeed, such possibilities are discussed in [48]. In such cases, we concede that the force of the above cases may be mitigated. However, such developments of the (A)-view are not our concern in this paper.

${ }^{40}$ In fact, the version of the Jacobson-Mattingly theory discussed in this paper is a special case of that presented in $[14,29]$.

${ }^{41}$ The first term is the Einstein-Hilbert action; $F_{a b}$ is the Faraday tensor associated to $A^{a}$ (see $\S 2$ ).
} 
frames aligned with that direction admit of further simplification, in the sense that further terms vanish in those frames. ${ }^{42}$ This notwithstanding, however, the form of the relativity principle presented in $\S 5$ is still satisfied, for the dynamical equations of this theory still take the same Poincaré-invariant form in all inertial frames.

Note that the dynamical equations of the Jacobson-Mattingly theory could be constructed via minimal coupling; ${ }^{43}$ this means that minimal coupling may lead to violations of certain forms of the relativity principle. In any case, however, the important point to make from the perspective of this section is that, in the Jacobson-Mattingly theory, the dynamical behaviour of non-gravitational fields does not reflect the local (Poincaré) symmetries of the metric fieldtaken to represent spacetime. The advocate of the (A)-view faces an outstanding burden to account for such cases.

Turn now to our second example: Bekenstein's bimetric TeVeS ('Tensor-Vector-Scalar') theory, presented in [3,4]. As discussed in [5, §9.5.2], in this theory the metric field which is surveyed by rods and clocks, the conformal structure of which is traced by light rays, and the geodesics of which correspond to the motion of free bodies, is not the 'fundamental' metric field $g_{a b}$, but rather a less 'fundamental' metric field $\tilde{g}_{a b}$, constructed from the other matter fields in the theory [5, p. 174]. Thus, one might claim that the TeVeS theory presents another case in which the local symmetries of the dynamical laws do not mirror the local (Poincaré) symmetries of the 'background' metric field-a necessary condition for the (A)-view to go through.

Still, in this case, both $g_{a b}$ and $\tilde{g}_{a b}$ are Lorentzian metric fields; moreover, the matter fields in this theory obey (in the relevant regime) locally Poincaré invariant dynamical laws. Thus, one might think that MR2 is satisfied in this case, and thus that the example does not raise problems for the (A)-view. What is going on here? In fact, $\mathrm{TeV} e \mathrm{~S}$ points to an interesting am-

\footnotetext{
${ }^{42}$ For an illustration of how this term leads to the breaking of local Lorentz invariance-and so violations of this form of the relativity principle - see the discussion of a somewhat simpler (but analogous) theory in appendix B. We do not discuss that simpler theory in the body of this paper, for it is a theory set in fixed spacetimewhereas our concern in the above is (in part) to focus upon theories with dynamical metric structure, which manifest (violations of) MR2. Nevertheless, it is worth noting that the theory presented in appendix B also raises questions regarding the kind of explanation for the dynamical behaviour of matter proffered by advocates of the geometrical approach. As a second point on the Jacobson-Mattingly theory, the Lagrange multiplier term in the Jacobson-Mattingly action also leads to a violation of gauge invariance-cf. [29, p. 3].

${ }^{43}$ For example, the dynamical equation $g_{a b} A^{a} A^{b}=1$ in the Jacobson-Mattingly theory could be constructed by applying the minimal coupling scheme to the associated dynamical equation $\eta_{a b} A^{a} A^{b}=1$.
} 
biguity in MR2. If this principle states that the local symmetry group of the dynamical laws governing matter fields is the same (in the relevant regime) as the local symmetry group of the metric field (in the sense given above), then it is indeed satisfied in TeVeS. Note, however, that in $\mathrm{TeV} e \mathrm{~S}$ it is the metric field $\tilde{g}_{a b}$, and not the metric field $g_{a b}$, which has chronogeometric significance-it is this field which takes a diagonal form in the frames in which the dynamical equations governing matter fields take their simplest form. If one reads MR2 as demanding this stronger condition (i.e., if one takes a strong reading of the word 'coincides' in MR2), then $\mathrm{TeV} e \mathrm{~S}$ violates this principle for the 'fundamental' metric field $g_{a b}$, for the local symmetries of $g_{a b}$ do not coincide in this strong sense with those of the dynamical laws governing matter fields. Since it is this stronger reading of MR2 which is relevant to a field's having chronogeometric significance, it is this reading which we prefer-in which case, $\mathrm{TeV} e \mathrm{~S}$ does pose a problem for the (A)-view, for it demonstrates that this local symmetry coincidence between the $g_{a b}$ field and the dynamical equations governing matter fields does not hold of necessity.

\section{Special Relativity}

With all the above in mind, we now reflect on the nature of SR, and its relation to GR. Results such as (2.12) indicate that Poincare invariance of dynamical laws in a spacetime theory is insufficient for that theory to be 'special relativistic', as such laws may still contain curvature pieces and derivatives of connection coefficients. Hence, some further criterion is needed to fully characterise 'special relativistic' theories. This criterion is that the inertial frames be global. To make sense of this, take the definition of an inertial frame presented in the previous section. As we have observed, $\Gamma_{\nu \rho}^{\mu}(p)=0$ in normal coordinates at $p$ (if the metriccompatible derivative operator is torsion-free), so this result holds in a local inertial frame. However, this is only generically true at $p$ : at neighbouring points the connection coefficients may be non-zero, i.e. partial derivatives of the connection components may be non-zero in normal coordinates at $p$; this leads to the possibility of curvature terms in higher order equations in local inertial frames.

What happens, however, if we now specify that the inertial frames are global? In that case, connection components vanish globally in normal coordinates, so it follows that par- 
tial derivatives of the connection coefficients also vanish in these frames. ${ }^{44}$ Hence, the Riemann curvature also vanishes globally. In such a case, higher order dynamical equations for non-gravitational fields in GR at any point $p \in M$ and written in normal coordinates take a Poincaré invariant form with no curvature terms, rather than merely a Poincare invariant form simpliciter. Moreover, this result holds at every point in spacetime. Without specifying that the inertial frames are global, we do not recover such dynamical equations, either globally or (in general) locally. Hence, one might argue that 'special relativistic' theories are best characterised through two criteria:

1. The dynamical laws governing matter fields are Poincaré invariant.

2. The inertial frames are global.

Deciding whether to characterise such theories via (1) and (2) together, or just via (1), is a cost-benefit analysis. In the former case, we retain the notion that SR concerns Poincaré invariant dynamical laws governing matter fields in a fixed Minkowski spacetime. ${ }^{45}$ However, in following this route we can no longer maintain that SR is in general recovered at a point in GR (although we can argue that it is approximately recovered, in the regime in which EP1' holds). By contrast, in the latter case we can maintain that SR is locally recovered in GR. However, one has to greatly expand one's conception of the scope of SR to include the study of the dynamics of matter fields in generically non-flat spacetimes.

Though it might seem that the 'safe' option here is to endorse the former of the two approaches, on which 'special relativistic' theories are characterised by both (1) and (2) (especially as (2) is, arguably, an essential conceptual assumption of SR-recall that one of Einstein's great insights on his quest towards GR was precisely the rejection of (2)), it is at least worth countenancing the latter, more general account, according to which a theory's being 'special relativistic' is tied to the Poincaré invariance of its dynamical laws alone, and no further a priori restriction is made on the content of these laws. On this view, the assumption of

\footnotetext{
${ }^{44}$ Note that we are not interested in the possibility of defining globally an arbitrary frame, but with the possibility that a given, globally-defined frame satisfies globally the condition of inertiality. Many thanks to an anonymous reviewer for helpful discussion on this point.

${ }^{45}$ Or at least, we almost do: an anonymous referee has pointed out that, in order to obtain Minkowski spacetime proper, we must specify that the manifold under consideration be diffeomorphic to $\mathbb{R}^{4}$, and geodesically complete.
} 
a fixed, background Minkowski spacetime is supererogatory to the core of SR. Indeed, if one does choose to characterise special relativistic theories through (1) alone, then, given certain restrictions on the form of dynamical laws for matter fields (e.g., that they be constructed via minimal coupling), GR itself is a locally special relativistic theory.

\section{Conclusions and Outlook}

There exist circumstances in GR in which minimal coupling leads to violations of EP1. In this paper, we have argued that this is unproblematic for advocates of the dynamical approach, for the relevant necessary condition for this view to account in the context of GR for the chronogeometricity of the dynamical metric field is, rather, the approximate validity of EP1', which obtains in the regime in which terms featuring the Riemann tensor or its contractions in dynamical equations for non-gravitational fields may be ignored. As a result, this position remains a live option for accounting for chronogeometricity in GR, when dynamical equations for non-gravitational fields are selected in accordance with minimal coupling.

Exploring the above themes led us to identify MR2: that, when terms representing tidal forces may be ignored, the symmetries of the dynamical laws for non-gravitational fields in the neighbourhood of any point $p \in M$ coincide with the symmetries of the primordial metric field in that neighbourhood. It appears, much as with MR1, that—absent some deeper storythis must simply be treated as a brute fact in GR. Though an explanation of minimal coupling, or certain forms of the relativity principle, would suffice to account for MR1 and MR2, no such explanation appears to be forthcoming. ${ }^{46}$ Though the advocate of the (A)-view might claim to be able to account for both of these 'miracles', we have presented two cases which call into question such assertions.

Finally, we have reflected on the nature of SR. While one might choose to characterise 'special relativistic' theories via two conditions-(i) that dynamical laws are Poincaré invariant, and (ii) that inertial frames are global-in this paper we have raised the possibility that such theories may be characterised solely by (i). Deciding which of these options is to be preferred is a nuanced business, worthy of future philosophical attention.

\footnotetext{
${ }^{46}$ At least within the domain of GR - it is possible that such an explanation can be found in e.g. perturbative string theory. See [50] for an exploration of this possibility.
} 


\section{Acknowledgements}

We are very grateful to Adam Caulton, Ricardo Heras, Tushar Menon, Wayne Myrvold, and Simon Saunders, for comments on earlier drafts of this paper; to three anonymous referees for assiduous and insightful feedback; as well as to Erik Curiel, Patrick Dürr, Domenico Giulini, Nick Huggett, Martin Lesourd, Niels Linnemann, Oliver Pooley, Nic Teh, and David Wallace, for valuable discussions. J.R. is supported by an AHRC studentship, and is also grateful to Hertford College for a graduate senior scholarship.

\section{A Minimal Coupling and Poincaré Invariance}

The goal of this appendix is to demonstrate that minimally coupled dynamical equations in GR manifest local Poincaré invariance, when written in normal coordinates at any $p \in M$. As a corollary, we derive some interesting results regarding when such equations exhibit a broader symmetry group. To begin, consider any minimally coupled dynamical equation in GR. In normal coordinates at some $p \in M$, this equation may schematically be written

$$
O_{1,1} \cdots O_{1, n_{1}}+\ldots+O_{m, 1} \cdots O_{m, n_{m}}=0
$$

Here, $m$ indexes the term; $n_{m}$ indexes the object in the $m$-th term. ${ }^{47}$ For such minimally coupled dynamical equations in normal coordinates, the set of relevant objects $O_{i}$ featuring in such equations consists of (a) tensors; (b) partial derivatives of tensors; and (c) partial derivatives of connection components. ${ }^{48}$ Given this, we now investigate the class of transformations under which (A.1) is invariant. ${ }^{49}$ Applying an arbitrary coordinate transformation to (A.1), we write each $O_{i}$ in the new, primed basis. Accordingly, our investigation reduces to an exploration of

\footnotetext{
${ }^{47}$ Indices in (A.1) are neither abstract nor coordinate indices, in the sense of the rest of this paper.

${ }^{48} \mathrm{We}$ do not include connection components in this enumeration, since these vanish in normal coordinates.

${ }^{49} \mathrm{~A}$ note on nomenclature. Consider an affine coordinate transformation $x^{\mu^{\prime}}=M^{\mu^{\prime}}{ }_{\mu} x^{\mu}+a^{\mu^{\prime}}$. If an $(r, s)$ tensor field $T^{\mu_{1} \ldots \mu_{r}}{ }_{\nu_{1} \ldots \nu_{s}}$ transforms under this coordinate change as $T_{\nu_{1} \ldots \nu_{s}}^{\mu_{1} \ldots \mu_{r}} \rightarrow$ $M_{\mu_{1}^{\prime}}^{\mu_{1}} \ldots M_{\mu_{r}^{\prime}}^{\mu_{r}} M_{\nu_{1}}^{\nu_{1}^{\prime}} \ldots M_{\nu_{s}}^{\nu_{s}^{\prime}} T_{1}^{\mu_{1}^{\prime} \ldots \mu_{r}^{\prime}} \nu_{1}^{\prime} \ldots \nu_{s}^{\prime}$, then we say that it is covariant with this coordinate transformation. If, on the other hand, a dynamical equation retains the same form in either of the two coordinate systems under consideration, then we say that it is invariant under the coordinate change.
} 
the individual transformation properties of each the $O_{i}$ in such minimally coupled equations, in normal coordinates. There are three cases to consider: (a)-(c). Suppose first (a): that $O_{i}$ is a tensor. Then, trivially, $O_{i}$ transforms as a tensor under arbitrary coordinate transformations. So now suppose (b): that the $O_{i}$ in question consists of a finite number of partial derivatives of a tensor. We claim that such an object transforms tensorially when the transformation in question is affine-i.e. when the transformation reads

$$
x^{\mu} \rightarrow x^{\mu^{\prime}}=M_{\mu}^{\mu^{\prime}} x^{\mu}+a^{\mu^{\prime}},
$$

where the matrix elements $M^{\mu^{\prime}}{ }_{\mu}$ and vector components $a^{\mu^{\prime}}$ are constant-in which case we have

$$
\frac{\partial x^{\mu^{\prime}}}{\partial x^{\mu}}=M_{\mu}^{\mu^{\prime}}
$$

To show this, proceed by induction. Base case: $O_{i}$ contains no partial derivatives of a tensor, so is just a tensor $\left(O_{i}=T_{\nu_{1} \ldots \nu_{s}}^{\mu_{1} \ldots \mu_{r}}\right)$. Then $O_{i}$ transforms tensorially under all coordinate transformations, a fortiori affine transformations. Inductive hypothesis: If $O_{i}$ consists of $n$ partial derivatives of a tensor, $O_{i}=\partial_{\sigma_{n}}^{(n)} \cdots \partial_{\sigma_{1}}^{(1)} T_{\nu_{1} \ldots \nu_{s}}^{\mu_{1} \ldots \mu_{r}}$, then $\partial_{\sigma_{(n-1)}}^{(n-1)} \cdots \partial_{\sigma_{1}}^{(1)} T_{\nu_{1} \ldots \nu_{s}}^{\mu_{1} \ldots \mu_{r}}$ transforms tensorially under affine coordinate transformations. Inductive step: For a given tensor $T^{\mu_{1} \ldots \mu_{r}}{ }_{\nu_{1} \ldots \nu_{s}}$, if $n$ partial derivatives of that tensor transforms tensorially under affine transformations, then show: so too does $O_{i}^{\prime}=\partial_{\sigma_{n+1}}^{(n+1)} O_{i}$. Proof: By the inductive hypothesis, $O_{i}=\partial_{\sigma_{n}}^{(n)} \cdots \partial_{\sigma_{1}}^{(1)} T_{\nu_{1} \ldots \nu_{s}}^{\mu_{1} \ldots \mu_{r}}$ transforms tensorially under an affine coordinate transformation:

$$
\begin{aligned}
O_{i} & =\partial_{\sigma_{n}}^{(n)} \cdots \partial_{\sigma_{1}}^{(1)} T_{\nu_{1} \ldots \nu_{s}}^{\mu_{1} \ldots \mu_{r}} \\
& \rightarrow M_{\sigma_{n}}^{\sigma_{n}^{\prime}} \cdots M_{\sigma_{1}}^{\sigma_{1}^{\prime}} M_{\mu_{1}^{\prime}}^{\mu_{1}} \cdots M_{\mu_{r}^{\prime}}^{\mu_{r}} M_{\nu_{1}}^{\nu_{1}^{\prime}} \cdots M_{\nu_{s}}^{\nu_{s}^{\prime}} \partial_{\sigma_{n}^{\prime}}^{(n)} \cdots \partial_{\sigma_{1}^{\prime}}^{(1)} T_{\nu_{1}^{\prime} \ldots \nu_{s}^{\prime}}^{\mu_{1}^{\prime} \ldots \mu_{\mu}^{\prime}},
\end{aligned}
$$

where the $M_{\mu^{\prime}}^{\mu}$ are as per (A.2). Given this, $O_{i}^{\prime}$ transforms as 


$$
\begin{aligned}
O_{i}^{\prime} \rightarrow M_{\sigma_{n+1}}^{\sigma_{n+1}^{\prime}} \partial_{\sigma_{n+1}^{\prime}}^{(n+1)}\left\{M_{\sigma_{n}}^{\sigma_{n}^{\prime}} \cdots M_{\sigma_{1}}^{\sigma_{1}^{\prime}} M_{\mu_{1}^{\prime}}^{\mu_{1}} \cdots M_{\mu_{r}^{\prime}}^{\mu_{r}}\right. \\
\left.M_{\nu_{1}}^{\nu_{1}^{\prime}} \cdots M_{\nu_{s}}^{\nu_{s}^{\prime}} \partial_{\sigma_{n}^{\prime}}^{(n)} \cdots \partial_{\sigma_{1}^{\prime}}^{(1)} T_{\nu_{1}^{\prime} \ldots \nu_{s}^{\prime}}^{\mu_{1}^{\prime} \ldots \mu_{r}^{\prime}}\right\}
\end{aligned}
$$

Distributing the partial derivative using the product rule, all terms of the form $\partial_{\sigma_{n+1}}^{(n+1)} M^{\lambda^{\prime}}$ vanish, leaving

$$
\begin{aligned}
O_{i}^{\prime} \rightarrow M_{\sigma_{n+1}}^{\sigma_{n+1}^{\prime}} M_{\sigma_{n}}^{\sigma_{n}^{\prime}} \cdots M_{\sigma_{1}}^{\sigma_{1}^{\prime}} M_{\mu_{1}^{\prime}}^{\mu_{1}} \cdots M_{\mu_{r}^{\prime}}^{\mu_{r}} . \\
M_{\nu_{1}}^{\nu_{1}^{\prime}} \cdots M_{\nu_{s}}^{\nu_{s}^{\prime}} \partial_{\sigma_{n+1}^{\prime}}^{(n+1)} \partial_{\sigma_{n}^{\prime}}^{(n)} \cdots \partial_{\sigma_{1}^{\prime}}^{(1)} T_{\nu_{1}^{\prime} \ldots \nu_{s}^{\prime}}^{\mu_{1}^{\prime} \ldots \mu_{r}^{\prime}}
\end{aligned}
$$

Hence, if the object $O_{i}$ consisting of $n$ partial derivatives of a tensor $T_{\nu_{1} \ldots \nu_{s}}^{\mu_{1} \ldots \mu_{r}}$ is invariant under affine coordinate transformations, then so too is the object $O_{i}^{\prime}$ consisting of $(n+1)$ partial derivatives of that same tensor. Thus, all partial derivatives of tensors appearing in minimally coupled dynamical equations at $p \in M$ transform tensorially under affine coordinate transformations.

Finally, consider (c). The claim now is that all partial derivatives of connection components transform tensorially under affine coordinate transformations. To prove this, again proceed by induction. Base case: $O_{i}=\partial_{\sigma} \Gamma_{\nu \lambda}^{\mu}$, i.e. $O_{i}$ consists of one partial derivative of a connection component. Transforming to a new coordinate basis and then expanding in terms of the old basis, we have 


$$
\begin{aligned}
\partial_{\rho^{\prime}} \Gamma_{\sigma^{\prime} \nu^{\prime}}^{\kappa^{\prime}=} & \frac{\partial x^{\rho}}{\partial x^{\rho^{\prime}}} \frac{\partial}{\partial x^{\rho}}\left(\frac{\partial x^{\sigma}}{\partial x^{\sigma^{\prime}}} \frac{\partial x^{\nu}}{\partial x^{\nu^{\prime}}} \Gamma_{\sigma \nu}^{\kappa} \frac{\partial x^{\kappa^{\prime}}}{\partial x^{\kappa}}+\frac{\partial x^{\kappa^{\prime}}}{\partial x^{\kappa}} \frac{\partial^{2} x^{\kappa}}{\partial x^{\sigma^{\prime}} \partial x^{\nu^{\prime}}}\right) \\
= & \frac{\partial^{2} x^{\sigma}}{\partial x^{\rho^{\prime}} \partial x^{\sigma^{\prime}}} \frac{\partial x^{\nu}}{\partial x^{\nu^{\prime}}} \Gamma_{\sigma \nu}^{\kappa} \frac{\partial x^{\kappa^{\prime}}}{\partial x^{\kappa}}+\frac{\partial x^{\sigma}}{\partial x^{\sigma^{\prime}}} \frac{\partial^{2} x^{\nu}}{\partial x^{\rho^{\prime}} \partial x^{\nu^{\prime}}} \Gamma_{\sigma \nu}^{\kappa} \frac{\partial x^{\kappa^{\prime}}}{\partial x^{\kappa}} \\
& +\frac{\partial x^{\rho}}{\partial x^{\rho^{\prime}}} \frac{\partial x^{\sigma}}{\partial x^{\sigma^{\prime}}} \frac{\partial x^{\nu}}{\partial x^{\nu^{\prime}}} \frac{\partial}{\partial x^{\rho}} \Gamma_{\sigma \nu}^{\kappa} \frac{\partial x^{\kappa^{\prime}}}{\partial x^{\kappa}}+\frac{\partial x^{\rho}}{\partial x^{\rho^{\prime}}} \frac{\partial x^{\sigma}}{\partial x^{\sigma^{\prime}}} \frac{\partial x^{\nu}}{\partial x^{\nu^{\prime}}} \Gamma_{\sigma \nu}^{\kappa} \frac{\partial^{2} x^{\kappa^{\prime}}}{\partial x^{\rho} \partial x^{\kappa}} \\
& +\frac{\partial x^{\rho}}{\partial x^{\rho^{\prime}}} \frac{\partial^{2} x^{\kappa^{\prime}}}{\partial x^{\rho} \partial x^{\kappa}} \frac{\partial^{2} x^{\kappa}}{\partial x^{\sigma^{\prime}} x^{\nu^{\prime}}}+\frac{\partial x^{\rho}}{\partial x^{\rho^{\prime}}} \frac{\partial x^{\kappa^{\prime}}}{\partial x^{\kappa}} \frac{\partial}{\partial x^{\rho}} \frac{\partial^{2} x^{\kappa}}{\partial x^{\sigma^{\prime}} \partial x^{\nu^{\prime}}} .
\end{aligned}
$$

Five of the six terms in (A.7) contain second partial derivative pieces, which vanish when the coordinate transformation is affine. So, under an affine coordinate transformation, this object transforms as a tensor. Inductive hypothesis: If $O_{i}$ consists of $n$ partial derivatives of a connection component, $O_{i}=\partial_{\sigma_{n}}^{(n)} \cdots \partial_{\sigma_{1}}^{(1)} \Gamma_{\nu \lambda}^{\mu}$, then $\partial_{\sigma_{(n-1)}}^{(n-1)} \cdots \partial_{\sigma_{1}}^{(1)} \Gamma_{\nu \lambda}^{\mu}$ transforms tensorially under affine coordinate transformations. Inductive step: If $O_{i}$ consists of $n$ partial derivatives of a connection component and transforms tensorially under affine transformations, then show: so too does $O_{i}^{\prime}=\partial_{\sigma_{n}+1}^{(n+1)} O_{i}$. Proof: mutatis mutandis as for the inductive step of (b). Thus, all partial derivatives of connection components appearing in minimally coupled dynamical equations at $p \in M$ transform tensorially under affine coordinate transformations.

We have found that each of the $O_{i}$ featuring in any minimally coupled dynamical equation in GR, written in normal coordinates at a point $p \in M$, is covariant-i.e., transforms tensorially-under affine coordinate transformations. However, we have yet to show that all such equations are invariant-i.e. take the same form-under affine coordinate transformations. In fact, this is in general not the case: and whether equations of the form (A.1) are so invariant, or rather are invariant under a restricted class of affine transformations (most relevantly the Poincaré group) depends upon context. To see this, first consider (2.9). Written in normal coordinates at some $p \in M$, the equation reads

$$
F_{[\mu \nu, \lambda]}=0
$$

Transforming to a new coordinate basis under an affine transformation, (A.8) reads, using the 
above results,

$$
M_{\mu}^{\mu^{\prime}} M_{\nu}^{\nu^{\prime}} M_{\lambda}^{\lambda^{\prime}} F_{\left[\mu^{\prime} \nu^{\prime}, \lambda^{\prime}\right]}=0
$$

At this stage, however, assuming that the transformation matrices are invertible, we may simply operate on (A.9) with the relevant matrix inverses, yielding

$$
F_{\left[\mu^{\prime} \nu^{\prime}, \lambda^{\prime}\right]}=0
$$

Hence, (2.9) written in normal coordinates at any $p \in M$ is invariant under all affine transformations with invertible linear transformation matrices. Now, however, consider (2.8). In normal coordinates at some $p \in M$, this reads

$$
F_{, \nu}^{\mu \nu}=J^{\mu}
$$

Transforming to a new coordinate basis under an affine transformation, (A.11) becomes

$$
M_{\mu^{\prime}}^{\mu} M_{\nu^{\prime}}^{\nu} M_{\nu}^{\lambda^{\prime}} F_{, \lambda^{\prime}}^{\mu^{\prime} \nu^{\prime}}=M_{\mu^{\prime}}^{\mu} M_{\nu^{\prime}}^{\nu} M_{\lambda^{\prime}}^{\sigma} \eta_{\nu \sigma} F^{\mu^{\prime} \nu^{\prime}, \lambda^{\prime}}=M_{\mu^{\prime}}^{\mu} J^{\mu^{\prime}}
$$

While the $M_{\mu^{\prime}}^{\mu}$ matrices can be cancelled (via multiplication by the relevant inverse matrices) in (A.12), in order for this equation to be invariant under the affine coordinate transformation in question, we require the further condition that

$$
M_{\nu^{\prime}}^{\nu} M_{\lambda^{\prime}}^{\sigma} \eta_{\nu \sigma}=\eta_{\nu^{\prime} \lambda^{\prime}}
$$

From this, we see that (2.8), written at some $p \in M$ in normal coordinates, is invariant only under the more restricted class of Poincaré transformations-since (A.13) is the definition of a Lorentz rotation. In general, whether the minimally coupled dynamical equations of matter fields in GR, written at a point in normal coordinates, are invariant under all affine transfor- 
mations (with invertible linear transformation matrices), or only the Poincare transformations, depends upon whether the metric field is used to contract indices between the $O_{i}$. To summarise:

- Objects of the form (a)-(c) transform tensorially under affine transformations; since the dynamical equations of GR obtained via minimal coupling, written at a point in normal coordinates, contain only objects of the form (a)-(c), all objects in such equations are covariant under affine transformations.

- Due to the potential contraction of indices in some terms of such equations with respect to the metric, we sometimes require further conditions on the affine transformations in question for that equation to be invariant thereunder. Where the metric is the Minkowski metric, this condition is that the transformations be Poincaré transformations.

The latter point is important. To illustrate, consider (2.1). We do not know under which affine transformations this equation is invariant until we specify the metric field with respect to which index contraction takes place. If this is the Minkowski metric, then such transformations are the Poincaré transformations, by the above reasoning. If, however, this is some other metric, then the affine transformations under which such objects are invariant will differ. ${ }^{50}$

\section{B Lorentz Symmetry Breaking}

Following [13, pp. 1231ff.], consider the Lagrangian for electrodynamics set in a fixed Minkowski spacetime as per $\S 2.1$, but now augmented with a Chern-Simons term, ${ }^{51}$

$$
\mathcal{L}_{\mathrm{CFJ}}=\mathcal{L}_{\mathrm{EM}}-\frac{1}{2} p_{a} A_{b} \tilde{F}^{a b}
$$

\footnotetext{
${ }^{50} \mathrm{Cf}$. [26,54]. As noted in [25,28], the specific spacetime metric implicit in one's theory of electrodynamics is specified by the so-called constitutive relations; for a certain simple form of the constitutive relations involving the Hodge dual operator, this is the Minkowski metric.

${ }^{51}$ For background on Chern-Simons theory, see e.g. [17]. Another theory which could be used to illustrate the same point is SR with a homogeneous dust field, the four-velocity field of which generates a timelike geodesic congruence. Our thanks to an anonymous reviewer for this point.
} 
Here, $\tilde{F}^{a b}:=\frac{1}{2} \epsilon^{a b c d} F_{c d}$ is the dual electromagnetic tensor. This modification couples electromagnetism to an (as yet unspecified) vector field, $p^{a} .{ }^{52}$ At the level of kinematically possible models, $p^{a}$ is specified to be spacelike, so that $p_{a} p^{a} \equiv m^{2}>0$. Varying the action associated to (B.1), one obtains a generalisation of (2.1),

$$
F_{; b}^{a b}=J^{a}+p_{b} \tilde{F}^{b a}
$$

by contrast, (2.2) still holds. (Note that $p_{a}:=\eta_{a b} p^{b}$.) Given that $p^{a}$ is spacelike, one can select a frame in which $p^{0}=0$, in which case (B.2) reduces to

$$
F_{; \mu}^{\nu \mu}=J^{\nu}+p_{i} \tilde{F}^{i \nu}
$$

Together, (B.3) and (2.2) $)^{53}$ are the simplest forms of the dynamical equations for this theory. The question, then, is: under which coordinate transformations are the forms of these equations preserved? Recalling from appendix A that (2.1) is invariant under Poincaré transformations, while (2.2) is invariant under all affine transformations with invertible linear transformation matrix, it is clear that the forms of (B.3) and (2.2) will together be invariant under the subgroup of Poincaré transformations that preserve the condition $p^{0}=0$. Our task now, then, is to identify this class of transformations.

By acting upon $p^{a}$ with an arbitrary transformation matrix, one finds that the coordinate transformations which preserve $p^{0}=0$ are the Galilean transformations. ${ }^{54}$ Thus, the class of transformations which preserve (B.3) and (2.2) together consists of the intersection of the Poincaré and Galilean transformations- these are the translations and spatial rotations. Thus, in this modified theory of electrodynamics, the presence of the $p^{a}$ vector field 'breaks' Lorentz

\footnotetext{
${ }^{52}$ As with the Minkowski metric field $\eta_{a b}$ in the version of electromagnetism introduced in $\$ 2.1$, the vector field $p^{a}$ may be understood to be a fixed field, in the sense of [45, p. 13].

${ }^{53}$ The latter written in a coordinate basis.

${ }^{54} \mathrm{To}$ see this, first act with a generic linear transformation matrix $M^{\mu}{ }_{\nu}$ (associated to an affine coordinate transformation $x^{\prime \mu}=M^{\mu}{ }_{\nu} x^{\nu}+a^{\mu}$-here we have moved the primes from indices to the new coordinates, i.e. have written $x^{\prime \mu}$ rather than $x^{\mu^{\prime}}$, for clarity of exposition) upon $p^{\mu}$ (this geometrical object now being written in some coordinate basis), subject to the condition that the temporal component also vanishes in the new coordinate system, i.e. ${p^{\prime}}^{0}=0$. Doing so, one finds that the time-space component $M_{i}^{0}$ of the transformation matrix vanishes. Given this, it follows that $x^{\prime 0}=M_{0}^{0} x^{0}+a^{0}$ and $x^{\prime i}=M_{0}^{i} x^{0}+M_{j}^{i} x^{j}+a^{i}$; these are precisely the Galilean transformations.
} 
symmetry, reducing the class of transformations under which the dynamical equations of the theory hold in their simplest form from the Poincaré transformations to the translations and spatial rotations. This is reconcilable with the Poincaré invariance of (B.2) and (2.2) together, since in this case these do not constitute the simplest forms of the dynamical equations of the theory. A similar result holds for the local dynamical equations at any $p \in M$ for the general relativistic Jacobson-Mattingly theory [14,29], discussed in $§ 6$.

\section{References}

[1] Pablo Acuña, "Minkowski Spacetime and Lorentz Invariance: The Cart and the Horse or Two Sides of a Single Coin?", Studies in History and Philosophy of Modern Physics 55, pp. 1-12, 2016.

[2] Yuri Balashov and Michel Janssen, "Presentism and Relativity", British Journal for the Philosophy of Science 54(2), pp. 327-346, 2003.

[3] Jacob D. Bekenstein, "An Alternative to the Dark Matter Paradigm: Relativistic MOND Gravitation", invited talk at the 28th Johns Hopkins Workshop on Current Problems in Particle Theory, June 2004, Johns Hopkins University, Baltimore. Available at arXiv:astro-ph/0412652.

[4] Jacob D. Bekenstein, "Relativistic Gravitation Theory for the MOND Paradigm", available at arXiv:astro-ph/0403694.

[5] Harvey R. Brown, Physical Relativity: Spacetime Structure from a Dynamical Perspective, Oxford: Oxford University Press, 2005.

[6] Harvey R. Brown and Peter Holland, "Simple Applications of Noether's First Theorem in Quantum Mechanics and Electromagnetism", American Journal of Physics 72, pp. 3439, 2004.

[7] Harvey R. Brown and Dennis Lehmkuhl, "Einstein, the Reality of Space, and the ActionReaction Principle", in Partha Ghose (ed.), Einstein, Tagore and the Nature of Reality, London and New York: Routledge, 2016. 
[8] Harvey R. Brown and Oliver Pooley, "The Origins of the Spacetime Metric: Bell's Lorentzian Pedagogy and its Significance in General Relativity", in Craig Callender and Nick Huggett (eds.), Physics Meets Philosophy at the Plank Scale, Cambridge: Cambridge University Press, 2001.

[9] Harvey R. Brown and Oliver Pooley, "Minkowski Space-Time: A Glorious Non-Entity", in Dennis Dieks (ed.), The Ontology of Spacetime, Elsevier, 2006.

[10] Harvey R. Brown and James Read, "Clarifying Possible Misconceptions in the Foundations of General Relativity", American Journal of Physics 84(5), pp. 327-334, 2016.

[11] Harvey R. Brown and James Read, "The Dynamical Approach to Spacetime Theories", in E. Knox and A. Wilson (eds.), The Routledge Companion to Philosophy of Physics, London: Routledge, 2018. (Forthcoming.)

[12] Craig Callender, What Makes Time Special?, Oxford: Oxford University Press, 2017.

[13] Sean Carroll, George Field and Roman Jackiw, "Limits on a Lorentz- and ParityViolating Modification of Electrodynamics”, Physical Review D 41(4), 1990.

[14] Sean Carroll and Eugene Lim, "Lorentz-Violating Vector Fields Slow the Universe Down”, Physical Review D 70, 123525, 2004.

[15] Erik Curiel, "On Geometric Objects, the Non-Existence of a Gravitational Stress-Energy Tensor, and the Uniqueness of the Einstein Field Equation", Studies in History and Philosophy of Modern Physics, 2017. (Forthcoming.)

[16] Neil Dewar and James Owen Weatherall, "On Gravitational Energy in Newtonian Theories", 2017.

[17] Gerald V. Dunne, "Aspects of Chern-Simons Theory", Les Houches Lectures, 1998. Available at arXiv:hep-th/9902115.

[18] Arthur Eddington, The Mathematical Theory of Relativity, Cambridge: Cambridge University Press, 1923.

[19] Albert Einstein, "On the Electrodynamics of Moving Bodies", Annalen der Physik 17, pp. 891-921, 1905. 
[20] Albert Einstein, "Die Grundlage der allgemeinen Relativitätstheorie", Annalen der Physik 49(7), pp. 769-822, 1916. Reprinted as Vol. 6, Doc. 30, CPAE.

[21] Albert Einstein, “The Fundaments of Theoretical Physics”, in Ideas and Opinions, New York: Bonanza, pp. 323-335, 1940.

[22] Michael Friedman, Foundations of Space-Time Theories, Princeton: Princeton University Press, 1983.

[23] Michel Ghins and Tim Budden, "The Principle of Equivalence”, Studies in History and Philosophy of Modern Physics 32, pp. 33-51, 2001.

[24] Hubert Goenner, "Theories of Gravitation with Nonminimal Coupling of Matter and the Gravitational Field”, Foundations of Physics 14(9), pp. 865-881, 1984.

[25] Friedrich Hehl and Yuri Obukhov, "How Does the Electromagnetic Field Couple to Gravity, in Particular to Metric, Nonmetricity, Torsion, and Curvature?", in Testing Relativistic Gravity in Space: Gyroscopes, Clocks, Interferometers, Bad Honnef, C. Laemmerzahl et al. (eds.). Springer, Berlin, 2000.

[26] José A. Heras, "Electromagnetism in Euclidean Four Space: A Discussion Between God and the Devil”, American Journal of Physics 62, pp. 914-916, 1994.

[27] Carl Hoefer, "Energy Conservation in GTR", Studies in History and Philosophy of Modern Physics 31, pp. 187-199, 2000.

[28] Yakov Itin and Friedrich Hehl, "Is the Lorentzian Signature of the Metric of Spacetime Electromagnetic in Origin?", Annals of Physics 312, pp. 60-83, 2004.

[29] Ted Jacobson and David Mattingly, "Gravity with a Dynamical Preferred Frame”, Physical Review D 64, 024028, 2001.

[30] Michel Janssen, "Drawing the Line Between Kinematics and Dynamics in Special Relativity", Studies in History and Philosophy of Modern Physics 40, pp. 26-52, 2009.

[31] Eleanor Knox, "Effective Spacetime Geometry", Studies in History and Philosophy of Modern Physics 44, pp. 346-356, 2013.

[32] Eleanor Knox, "Newtonian Spacetime Structure in Light of the Equivalence Principle", British Journal for the Philosophy of Science 65(4), pp. 863-880, 2014. 
[33] Vincent Lam, "Gravitational and Nongravitational Energy: The Need for Background Structures", Philosophy of Science 78, pp. 1012-1024, 2011.

[34] A. R. Lee and T. M. Kalotas, "Lorentz Transformations from the First Postulate", American Journal of Physics 43, pp. 434-437, 1975.

[35] Dennis Lehmkuhl, "Mass-Energy-Momentum: Only there Because of Spacetime?", British Journalfor the Philosophy of Science 62, 2011.

[36] Dennis Lehmkuhl, "The Equivalence Principle(s)", forthcoming in E. Knox and A. Wilson (eds.), The Routledge Companion to the Philosophy of Physics, London: Routledge, 2018.

[37] J.-M. Lévy-Leblond, "One More Derivation of the Lorentz Transformation", American Journal of Physics 44, pp. 271-279, 1976.

[38] David Malament, Topics in the Foundations of General Relativity and Newtonian Gravitation Theory, Chicago: University of Chicago Press, 2012.

[39] Tim Maudlin, Philosophy of Physics Volume I: Space and Time, Princeton: Princeton University Press, 2012.

[40] Charles Misner, Kip Thorne, and John Wheeler, Gravitation, San Francisco: Freeman \& Co., 1973.

[41] John Norton, "What Was Einstein's Principle of Equivalence?", Studies in History and Philosophy of Science 16, pp. 203-246, 1985.

[42] Hans C. Ohanian, "What is the Principle of Equivalence?", American Journal of Physics 45, pp. 903-909, 1977.

[43] Hans C. Ohanian and Remo Ruffini, Gravitation and Spacetime, New York: W. W. Norton \& Co., 1994.

[44] Oliver Pooley, "Substantivalist and Relationist Approaches to Spacetime", in R. Batterman (ed.), The Oxford Handbook of Philosophy of Physics, Oxford University Press, 2013. 
[45] Oliver Pooley, "Background Independence, Diffeomorphism Invariance, and the Meaning of Coordinates", in D. Lehmkuhl, G. Schiemann and E. Scholz (eds.), Towards a Theory of Spacetime Theories, Birkhäuser, 2017.

[46] Eric Poisson and Clifford M. Will, Gravity: Newtonian, Post-Newtonian, Relativistic, Cambridge: Cambridge University Press, 2014.

[47] R. V. Pound and G. A. Rebka Jr., "Apparent Weight of Photons", Physical Review Letters 4(7), pp. 337-341, 1960.

[48] James Read, "Explanation, Geometry, and Conspiracy in Relativity Theory”, 2017.

[49] James Read, "Functional Gravitational Energy", British Journal for the Philosophy of Science, 2017. (Forthcoming.)

[50] James Read, "No Miracles in String Theory”, 2017.

[51] Erwin Schrödinger, Space-Time Structure, Cambridge: Cambridge University Press, 1950.

[52] David Wallace, "Who's Afraid of Coordinate Systems? An Essay on Representation of Spacetime Structure", Studies in History and Philosophy of Modern Physics, 2017. (Forthcoming.)

[53] James Owen Weatherall, "On (Some) Explanations in Physics", Philosophy of Science 78(3), pp. 421-447, 2011.

[54] E. Zampino, "A Brief Study on the Transformation of Maxwell Equations in Euclidean Four-Space", Journal of Mathematical Physics 27, pp. 1315-1318, 1986. 